\title{
A ČECH TYPE CONSTRUCTION FOR EQUIVARIANT COHOMOLOGY
}

\author{
Hannu Honkasalo
}

\section{Introduction}

A classical result of Dowker [D] states that non-equivariant Alexander-Spanier cohomology is isomorphic to Cech cohomology on arbitrary spaces. In $[\mathrm{H}]$ we constructed an equivariant version of Alexander-Spanier cohomology, defined for all $G$-pairs, $G$ a finite group. The purpose of this paper is to give a suitable equivariant generalization of Cech cohomology, and then to prove the appropriate generalization of the above mentioned result of Dowker.

The contents of the paper are as follows: In Section 1 we present the construction of the equivariant Cech cohomology groups and state the main result about the isomorphism between equivariant Alexander-Spanier and Čech cohomology. The proof of this result generalizes the method outlined in [S], exercises 6 D 1-3, for proving the theorem of Dowker. Our proof occupies Sections 2 and 3 below: in Section 2 we show that it is enough to find chain homotopy equivalences between certain chain complexes of contravariant coefficient systems, and in Section 3 we carry out the construction of such homotopy equivalences by the method of acyclic models. In this paper we have to use a slightly different definition of equivariant Alexander-Spanier cohomology than the one we used in $[\mathrm{H}]$. We ponder this difference in an appendix.

\section{The construction and main result}

Let $G$ be a finite group; this hypothesis holds in all that follows. As in [B], let $\mathcal{O}_{G}$ denote the category of the canonical $G$-orbits $G / H, H \leq G$, and $\mathcal{C}_{G}$ the category of contravariant coefficient systems, i.e. contravariant functors $\mathcal{O}_{G} \rightarrow A b$.

Let $X$ be a $G$-space and $A \subset X$ a $G$-subspace; we use the convention that the definition of a $G$-space includes the Hausdorff condition. In the following, a $G$-covering of $X$ means an open covering $\mathcal{U}=\left\{U_{x} \mid x \in X\right\}$ of $X$ satisfying

$$
x \in U_{x} \quad \text { for each } x \in X, \quad g U_{x}=U_{g x} \quad \text { for each } x \in X, g \in G
$$

(here we follow an idea of Godement, cf. [G], p. 223). Given such a $\mathcal{U}$, we form the $G$-covering $\mathcal{U}^{\prime}=\left\{U_{x} \cap A \mid x \in A\right\}$ of $A$. 
Let $\mathcal{U}$ be a $G$-covering of $X$, as above. For each $n \in \mathbf{N}$ we define a coefficient system $\underline{C}_{n}(\mathcal{U}) \in \mathcal{C}_{G}$ by

$$
\begin{aligned}
& \underline{C}_{n}(\mathcal{U}): G / H \mapsto \text { free abelian group with basis } \\
& \quad\left\{\left(x_{0}, \ldots, x_{n}\right) \in\left(X^{H}\right)^{n+1} \mid U_{x_{0}} \cap \cdots \cap U_{x_{n}} \cap X^{H} \neq \emptyset\right\}
\end{aligned}
$$

for $H \leq G . \underline{C}_{n}(\mathcal{U})$ has obvious values on morphisms of $\mathcal{O}_{G}$. Namely, a morphism $\varphi: G / K \rightarrow G / H$ has the form $g K \mapsto g a H$, where $a^{-1} K a \leq H$, and we set $\underline{C}_{n}(\mathcal{U})(\varphi):\left(x_{0}, \ldots, x_{n}\right) \mapsto\left(a x_{0}, \ldots, a x_{n}\right)$; this is well-defined, because

$$
\begin{aligned}
U_{a x_{0}} & \cap \cdots \cap U_{a x_{n}} \cap X^{K} \supset U_{a x_{0}} \cap \cdots \cap U_{a x_{n}} \cap X^{a H a^{-1}} \\
& =a \cdot\left(U_{x_{0}} \cap \cdots \cap U_{x_{n}} \cap X^{H}\right)
\end{aligned}
$$

is non-empty, if $U_{x_{0}} \cap \cdots \cap U_{x_{n}} \cap X^{H} \neq \emptyset$. There are obvious boundary morphisms $\partial: \underline{C}_{n}(\mathcal{U}) \rightarrow \underline{C}_{n-1}(\mathcal{U})$ in $\mathcal{C}_{G}(n \geq 1)$ determined by $\left(x_{0}, \ldots, x_{n}\right) \mapsto$ $\sum_{i=0}^{n}(-1)^{i}\left(x_{0}, \ldots, \hat{x}_{i}, \ldots, x_{n}\right)$, and hence $\underline{C}_{*}(\mathcal{U})$ is a chain complex in $\mathcal{C}_{G}$.

The preceding construction applied to the $G$-covering $\mathcal{U}^{\prime}$ of $A$ yields the chain complex $\underline{C}_{*}\left(\mathcal{U}^{\prime}\right)$. Because $\underline{C}_{*}\left(\mathcal{U}^{\prime}\right)(G / H) \subset \underline{C}_{*}(\mathcal{U})(G / H)$ for each $H \leq G$, there is canonical monomorphism $\underline{C}_{*}\left(\mathcal{U}^{\prime}\right) \hookrightarrow \underline{C}_{*}(\mathcal{U})$. We define a chain complex $\underline{C}_{*}\left(\mathcal{U}, \mathcal{U}^{\prime}\right)$ in $\mathcal{C}_{G}$ by

$$
\underline{C}_{*}\left(\mathcal{U}, \mathcal{U}^{\prime}\right)=\operatorname{coker}\left[\underline{C}_{*}\left(\mathcal{U}^{\prime}\right) \hookrightarrow \underline{C}_{*}(\mathcal{U})\right] .
$$

We call a $G$-covering $\mathcal{V}$ of $X$ a refinement of $\mathcal{U}$ provided that $V_{x} \subset U_{x}$ for each $x \in X$. If $\mathcal{V}$ is a refinement of $\mathcal{U}$, there is a canonical monomorphism $\underline{C}_{*}\left(\mathcal{V}, \mathcal{V}^{\prime}\right) \rightarrow \underline{C}_{*}\left(\mathcal{U}, \mathcal{U}^{\prime}\right)$, which induces a cochain map $\operatorname{Hom}_{\mathcal{C}_{G}}\left(\underline{C}_{*}\left(\mathcal{U}, \mathcal{U}^{\prime}\right), m\right) \rightarrow$ $\operatorname{Hom}_{\mathcal{C}_{G}}\left(\underline{C}_{*}\left(\mathcal{V}, \mathcal{V}^{\prime}\right), m\right)$ for any $m \in \mathcal{C}_{G}$ (here it is essential that we use only $G$ coverings of the particular kind specified above).

Definition 1.1. Let $m \in \mathcal{C}_{G}$ be a contravariant coefficient system. The equivariant Čech cochain complex of $(X, A)$ with coefficients $m$ is

$$
\check{C}_{G}^{*}(X, A ; m)=\underset{\mathcal{U}}{\lim } \operatorname{Hom}_{\mathcal{C}_{G}}\left(\underline{C}_{*}\left(\mathcal{U}, \mathcal{U}^{\prime}\right), m\right) .
$$

The equivariant $\check{C}$ ch cohomology groups of $(X, A)$ with coefficients $m$ are

$$
\check{H}_{G}^{n}(X, A ; m)=H^{n}\left(\check{C}_{G}^{*}(X, A ; m)\right), \quad n \in \mathbf{N} .
$$

Next we give a description of the equivariant Alexander-Spanier cohomology of $(X, A)$, which differs slightly from the definition given in [H]. If $\mathcal{U}$ is a $G$ covering of $X$, we can define chain complexes $\underline{D}_{*}(\mathcal{U}), \underline{D}_{*}\left(\mathcal{U}^{\prime}\right)$ and $\underline{D}_{*}\left(\mathcal{U}, \mathcal{U}^{\prime}\right)$ in $\mathcal{C}_{G}$ such that

$\underline{D}_{n}(\mathcal{U}): G / H \mapsto$ free abelian group with basis $\left\{\left(y_{0}, \ldots, y_{n}\right) \in\left(X^{H}\right)^{n+1} \mid\left\{y_{0}, \ldots, y_{n}\right\} \subset U_{x}\right.$ for some $\left.x \in X^{H}\right\}$, 


$$
\underline{D}_{*}\left(\mathcal{U}, \mathcal{U}^{\prime}\right)=\operatorname{coker}\left[\underline{D}_{*}\left(\mathcal{U}^{\prime}\right) \hookrightarrow \underline{D}_{*}(\mathcal{U})\right]
$$

Proposition 1.2. For any coeffcient system $m \in \mathcal{C}_{G}$,

$$
\underset{\mathfrak{U}}{\lim _{\longrightarrow}} \operatorname{Hom}_{\mathcal{C}_{G}}\left(\underline{D}_{*}\left(\mathcal{U}, \mathcal{U}^{\prime}\right), m\right)
$$

is isomorphic to the equivariant Alexander-Spanier cochain complex $\bar{C}_{G}^{*}(X, A ; m)$ as defined in $[\mathrm{H}]$.

Proof. Cf. the appendix. 口

The main result of this paper is the following theorem which will be proved in Sections 2 and 3, as promised in the Introduction:

Theorem 1.3. There is a natural isomorphism $\check{H}_{G}^{*}(X, A ; m) \cong \bar{H}_{G}^{*}(X, A ; m)$ between the equivariant Čech and Alexander-Spanier cohomology of $(X, A)$ with arbitrary coefficients $m \in \mathcal{C}_{G}$.

\section{Subdivision}

Let $(X, A)$ be a $G$-pair and $\mathcal{U}=\left\{U_{x} \mid x \in X\right\}$ a $G$-covering of $X$, as above. If $H \leq G$, we denote $\mathcal{U}_{H}=\left\{U_{x} \cap X^{H} \mid x \in X^{H}\right\}$, an open covering of $X^{H}$. Let $K\left(\mathcal{U}_{H}\right)$ be the nerve of $\mathcal{U}_{H}$, i.e., $K\left(\mathcal{U}_{H}\right)$ is a simplicial complex, a simplex of $K\left(\mathcal{U}_{H}\right)$ being a finite subset $\left\{x_{0}, \ldots, x_{n}\right\} \subset X^{H}$ satisfying $U_{x_{0}} \cap \cdots \cap$ $U_{x_{n}} \cap X^{H} \neq \emptyset$. Also, let $L\left(\mathcal{U}_{H}\right)$ be the simplicial complex, whose simplexes are those finite subsets $\left\{y_{0}, \ldots, y_{m}\right\} \subset X^{H}$ which satisfy $\left\{y_{0}, \ldots, y_{m}\right\} \subset U_{x}$ for some $x \in X^{H}$. We remark that a $G$-map $G / H_{1} \rightarrow G / H_{2}$ induces obvious simplicial maps $K\left(\mathcal{U}_{H_{2}}\right) \rightarrow K\left(\mathcal{U}_{H_{1}}\right)$ and $L\left(\mathcal{U}_{H_{2}}\right) \rightarrow L\left(\mathcal{U}_{H_{1}}\right)$. In this notation we have

$$
\begin{aligned}
\underline{C}_{*}(\mathcal{U}): G / H & \mapsto C_{*}\left(K\left(\mathcal{U}_{H}\right)\right) \\
\underline{C}_{*}\left(\mathcal{U}^{\prime}\right): G / H & \mapsto C_{*}\left(K\left(\mathcal{U}_{H}^{\prime}\right)\right) \\
\underline{C}_{*}\left(\mathcal{U}, \mathcal{U}^{\prime}\right): G / H & \mapsto C_{*}\left(K\left(\mathcal{U}_{H}\right), K\left(\mathcal{U}_{H}^{\prime}\right)\right) \\
\underline{D}_{*}(\mathcal{U}): G / H & \mapsto C_{*}\left(L\left(\mathcal{U}_{H}\right)\right) \\
\underline{D}_{*}\left(\mathcal{U}^{\prime}\right): G / H & \mapsto C_{*}\left(L\left(\mathcal{U}_{H}^{\prime}\right)\right) \\
\underline{D}_{*}\left(\mathcal{U}, \mathcal{U}^{\prime}\right): G / H & \mapsto C_{*}\left(L\left(\mathcal{U}_{H}\right), L\left(\mathcal{U}_{H}^{\prime}\right)\right)
\end{aligned}
$$

for any $H \leq G$, where $C_{*}(K)$ means the ordered chain complex of the simplicial complex $K$.

We recall briefly the definition of the barycentric subdivision $\operatorname{Sd} K$ of an abstract simplicial complex $K$. The vertexes of $\operatorname{Sd} K$ are the simplexes of $K$, and a finite set of simplexes of $K$ is a simplex of $\operatorname{Sd} K$ if it is linearly ordered by inclusion. Recall also that there is a natural subdivision chain map Sd: $C_{*}(K) \rightarrow$ $C_{*}(\mathrm{Sd} K)$, which is a chain homotopy equivalence for any $K$ (cf. [E-St], VI 8). 
We write $K^{\prime}\left(\mathcal{U}_{H}\right)=\operatorname{Sd} K\left(\mathcal{U}_{H}\right), L^{\prime}\left(\mathcal{U}_{H}\right)=\operatorname{Sd} L\left(\mathcal{U}_{H}\right)$ etc. $(H \leq G)$. We can now define the following new chain complexes of $\mathcal{C}_{G}$ :

$$
\begin{aligned}
\underline{C}_{*}^{\prime}(\mathcal{U}): G / H & \mapsto C_{*}\left(K^{\prime}\left(\mathcal{U}_{H}\right)\right) \\
\underline{C}_{*}^{\prime}\left(\mathcal{U}^{\prime}\right): G / H & \mapsto C_{*}\left(K^{\prime}\left(\mathcal{U}_{H}^{\prime}\right)\right) \\
\underline{C}_{*}^{\prime}\left(\mathcal{U}, \mathcal{U}^{\prime}\right): G / H & \mapsto C_{*}\left(K^{\prime}\left(\mathcal{U}_{H}\right), K^{\prime}\left(\mathcal{U}_{H}^{\prime}\right)\right) \\
\underline{D}_{*}^{\prime}(\mathcal{U}): G / H & \mapsto C_{*}\left(L^{\prime}\left(\mathcal{U}_{H}\right)\right) \\
\underline{D}_{*}^{\prime}\left(\mathcal{U}^{\prime}\right): G / H & \mapsto C_{*}\left(L^{\prime}\left(\mathcal{U}_{H}^{\prime}\right)\right) \\
\underline{D}_{*}^{\prime}\left(\mathcal{U}, \mathcal{U}^{\prime}\right): G / H & \mapsto C_{*}\left(L^{\prime}\left(\mathcal{U}_{H}\right), L^{\prime}\left(\mathcal{U}_{H}^{\prime}\right)\right)
\end{aligned}
$$

with obvious values on morphisms of $\mathcal{O}_{G}$. The above mentioned subdivision chain maps, being natural, define the following chain maps in $\mathcal{C}_{G}:$ sd $: \underline{C}_{*}(\mathcal{U}) \rightarrow \underline{C}_{*}^{\prime}(\mathcal{U})$, sd : $\underline{C}_{*}\left(\mathcal{U}^{\prime}\right) \rightarrow \underline{C}_{*}^{\prime}\left(\mathcal{U}^{\prime}\right)$, sd $: \underline{C}_{*}\left(\mathcal{U}, \mathcal{U}^{\prime}\right) \rightarrow \underline{C}_{*}^{\prime}\left(\mathcal{U}, \mathcal{U}^{\prime}\right)$, and similarly with $\underline{C}_{*}$ replaced by $\underline{D}_{*}$.

If $\mathcal{U}$ is a refinement of a $G$-covering $\mathcal{V}=\left\{V_{x} \mid x \in X\right\}$, there are evident chain maps $\underline{C}_{*}^{\prime}\left(\mathcal{U}, \mathcal{U}^{\prime}\right) \rightarrow \underline{C}_{*}^{\prime}\left(\mathcal{V}, \mathcal{V}^{\prime}\right)$ and $\underline{D}_{*}^{\prime}\left(\mathcal{U}, \mathcal{U}^{\prime}\right) \rightarrow \underline{D}_{*}^{\prime}\left(\mathcal{V}, \mathcal{V}^{\prime}\right)$.

Lemma 2.3. The subdivision chain maps are compatible with refinement, i.e., if $\mathcal{U}$ is a refinement of $\mathcal{V}$, then the squares

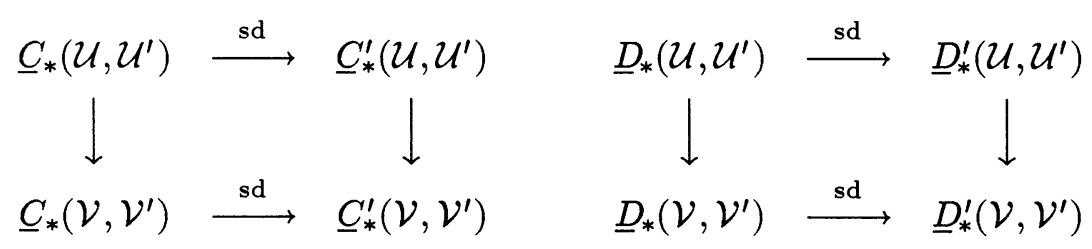

commute.

Proof. Taking values at $G / H(H \leq G)$ we obtain two squares, where the vertical arrows are induced by certain simplicial maps $\left(K\left(\mathcal{U}_{H}\right), K\left(\mathcal{U}_{H}^{\prime}\right)\right) \rightarrow$ $\left(K\left(\mathcal{V}_{H}\right), K\left(\mathcal{V}_{H}^{\prime}\right)\right)$ and $\left(L\left(\mathcal{U}_{H}\right), L\left(\mathcal{U}_{H}^{\prime}\right)\right) \rightarrow\left(L\left(\mathcal{V}_{H}\right), L\left(\mathcal{V}_{H}^{\prime}\right)\right)$. The commutativity thus follows from the naturality of sd with respect to simplicial maps. $\square$

The next two results, to be proved in Section 3, are main ingredients in the proof of Theorem 1.3:

Proposition 2.4. The chain maps

$$
\text { sd: } \underline{C}_{*}\left(\mathcal{U}, \mathcal{U}^{\prime}\right) \rightarrow \underline{C}_{*}^{\prime}\left(\mathcal{U}, \mathcal{U}^{\prime}\right)
$$

and

$$
\text { sd: } \underline{D}_{*}\left(\mathcal{U}, \mathcal{U}^{\prime}\right) \rightarrow \underline{D}_{*}^{\prime}\left(\mathcal{U}, \mathcal{U}^{\prime}\right)
$$

are chain homotopy equivalences for any $G$-covering $\mathcal{U}$ of $X$. 
Proposition 2.5. For each $G$-covering $\mathcal{U}$ of $X$ there is a chain homotopy equivalence $\alpha: \underline{C}_{*}^{\prime}\left(\mathcal{U}, \mathcal{U}^{\prime}\right) \rightarrow \underline{D}_{*}^{\prime}\left(\mathcal{U}, \mathcal{U}^{\prime}\right)$ in $\mathcal{C}_{G}$. The equivalences $\alpha$ for various $\mathcal{U}$ can be chosen in such a way that the square

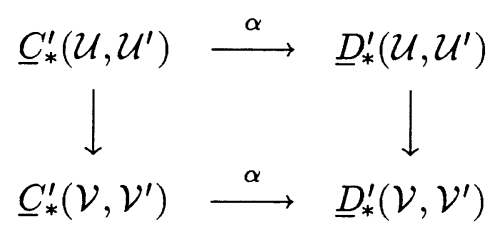

commutes up to chain homotopy, whenever $\mathcal{U}$ is a refinement of $\mathcal{V}$ (i.e. $\alpha$ is compatible with refinement up to chain homotopy).

The proof of Theorem 1.3 is immediate, once we have proved 2.4 and 2.5 . Namely, 2.4 implies that, for $n \in \mathbf{N}$,

$$
\begin{aligned}
& \check{H}_{G}^{n}(X, A ; m) \cong \underset{\lim }{\vec{u}} H^{n}\left(\operatorname{Hom}_{\mathcal{C}_{G}}\left(\underline{C}_{*}^{\prime}\left(\mathcal{U}, \mathcal{U}^{\prime}\right), m\right)\right), \\
& \bar{H}_{G}^{n}(X, A ; m) \cong \underset{\mathcal{u}}{\underline{u}} H^{n}\left(\operatorname{Hom}_{\mathcal{C}_{G}}\left(\underline{D}_{*}^{\prime}\left(\mathcal{U}, \mathcal{U}^{\prime}\right), m\right)\right)
\end{aligned}
$$

and the chain homotopy equivalences $\alpha$ of 2.5 induce the required isomorphism between these groups.

\section{Construction of the chain maps and homotopies}

Let $\mathcal{U}$ be a covering of $X$ as before. We define the following four categories:

$$
\begin{aligned}
\text { category } & \text { objects } \\
\mathcal{C}_{\mathcal{U}} & \text { subcomplexes } K \text { of } K\left(\mathcal{U}_{H}\right) \text { or } K\left(\mathcal{U}_{H}^{\prime}\right), \quad H \leq G \\
\mathcal{C}_{\mathcal{U}}^{\prime} & \text { subcomplexes } K^{\prime} \text { of } K^{\prime}\left(\mathcal{U}_{H}\right) \text { or } K^{\prime}\left(\mathcal{U}_{H}^{\prime}\right), \quad H \leq G, \\
\mathcal{D}_{\mathcal{U}} & \text { subcomplexes } L \text { of } L\left(\mathcal{U}_{H}\right) \text { or } L\left(\mathcal{U}_{H}^{\prime}\right), \quad H \leq G \\
\mathcal{C}_{\mathcal{U}}^{\prime} & \text { subcomplexes } L^{\prime} \text { of } L^{\prime}\left(\mathcal{U}_{H}\right) \text { or } L^{\prime}\left(\mathcal{U}_{H}^{\prime}\right), \quad H \leq G
\end{aligned}
$$

here subcomplexes of $K\left(\mathcal{U}_{H}\right)$ and $K\left(\mathcal{U}_{H}^{\prime}\right)$, as well as those of $K\left(\mathcal{U}_{H_{1}}\right)$ and $K\left(\mathcal{U}_{H_{2}}\right)$ for $H_{1} \neq H_{2}$, are considered distinct, and similarly in the other three cases. In each of the four categories, a morphism is a simplicial embedding induced by some $a \in G$ (for example, a morphism of $\mathcal{C}_{\mathcal{U}}$ is given by $x \mapsto a x$ on vertexes).

Let $C^{a}(A b)$ be the category of augmented chain complexes of abelian groups. Next we define the functors appearing in the diagram below: 


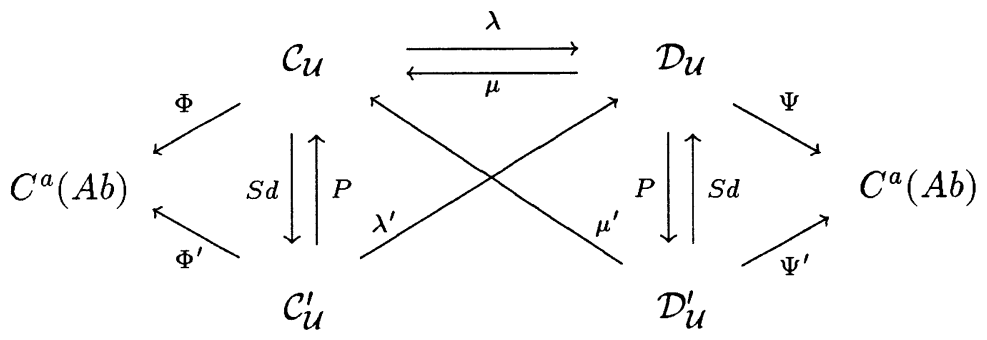

Each of the functors $\Phi, \Phi^{\prime}, \Psi$ and $\Psi^{\prime}$ associates to a simplicial complex its ordered chain complex (for example, if $K \in \mathcal{C}_{\mathcal{U}}$, then $\Phi(K)=C_{*}(K)$ ). The functors Sd associate to a simplicial complex its barycentric subdivision.

(The alert reader may notice that for example the chain complex $\Phi(K)$ is augmented over $\mathbf{Z}$ only if $K \neq \emptyset$. It is, however, easy to see that this slight ambiguity does not affect the use of the acyclic model theorem in what follows.)

To define $P: \mathcal{C}_{\mathcal{U}}^{\prime} \rightarrow \mathcal{C}_{\mathcal{U}}$, let $s$ be a vertex of $K^{\prime}\left(\mathcal{U}_{H}\right)$ (or $K^{\prime}\left(\mathcal{U}_{H}^{\prime}\right)$ ), $H \leq G$, i.e. $s$ is a simplex of $K\left(\mathcal{U}_{H}\right)$ (or $K\left(\mathcal{U}_{H}^{\prime}\right)$ ). We set $P(s)=\bar{s}$, the subcomplex of $K\left(\mathcal{U}_{H}\right)$ (or $K\left(\mathcal{U}_{H}^{\prime}\right)$ ) consisting of the faces of $s$. If $K^{\prime} \in \mathcal{C}_{\mathcal{U}}^{\prime}$, i.e. $K^{\prime}$ is a subcomplex of $K^{\prime}\left(\mathcal{U}_{H}\right)$ (or $K^{\prime}\left(\mathcal{U}_{H}^{\prime}\right)$ ), we let

$$
P\left(K^{\prime}\right)=\bigcup\left\{P(s) \mid s \in K^{\prime} \text { vertex }\right\}=\bigcup\left\{\bar{s} \mid s \in K^{\prime} \text { vertex }\right\} .
$$

The values of $P: \mathcal{D}_{\mathcal{U}}^{\prime} \rightarrow \mathcal{D}_{\mathcal{U}}$ are defined similarly.

If $s=\left\{x_{0}, \ldots, x_{n}\right\}$ is again a simplex of $K\left(\mathcal{U}_{H}\right)$ (or $K\left(\mathcal{U}_{H}^{\prime}\right)$ ), i.e. a vertex of $K^{\prime}\left(\mathcal{U}_{H}\right)$ (or $\left.K^{\prime}\left(\mathcal{U}_{H}^{\prime}\right)\right), H \leq G$, let $\lambda(s)$ be the subcomplex of $L\left(\mathcal{U}_{H}\right)$ (or $L\left(\mathcal{U}_{H}^{\prime}\right)$ ) consisting of all simplexes $t \subset U_{x_{0}} \cap \cdots \cap U_{x_{n}}$. If $K \in \mathcal{C}_{\mathcal{U}}$ and $K^{\prime} \in \mathcal{C}_{\mathcal{U}}^{\prime}$, define

$$
\begin{aligned}
\lambda(K) & =\bigcup\{\lambda(x) \mid x \in K \text { vertex }\}, \\
\lambda^{\prime}\left(K^{\prime}\right) & =\bigcup\left\{\lambda(s) \mid s \in K^{\prime} \text { vertex }\right\} .
\end{aligned}
$$

This defines $\lambda$ and $\lambda^{\prime}$ in 3.2 .

If $t$ is a simplex of $L\left(\mathcal{U}_{H}\right)$ (or $L\left(\mathcal{U}_{H}^{\prime}\right)$ ), i.e. a vertex of $L^{\prime}\left(\mathcal{U}_{H}\right)$ (or $L^{\prime}\left(\mathcal{U}_{H}^{\prime}\right)$ ), $H \leq G$, let $\mu(t)$ be the subcomplex of $K\left(\mathcal{U}_{H}\right)$ (or $K\left(\mathcal{U}_{H}^{\prime}\right)$ ) consisting of all simplexes $\left\{x_{0}, \ldots, x_{n}\right\}$ such that $t \subset U_{x_{0}} \cap \cdots \cap U_{x_{n}}$. If $L \in \mathcal{D}_{\mathcal{U}}$ and $L^{\prime} \in \mathcal{D}_{\mathcal{U}}^{\prime}$, define

$$
\begin{aligned}
\mu(L) & =\bigcup\{\mu(y) \mid y \in L \text { vertex }\}, \\
\mu^{\prime}\left(L^{\prime}\right) & =\bigcup\left\{\mu(t) \mid t \in L^{\prime} \text { vertex }\right\} .
\end{aligned}
$$

This completes the construction of diagram 3.2. 
Subdivision chain maps determine natural transformations

$$
\text { sd: } \Phi \rightarrow \Phi^{\prime} \circ \mathrm{Sd}, \quad \text { sd: } \Psi \rightarrow \Psi^{\prime} \circ \mathrm{Sd} .
$$

Furthermore, because every vertex of a simplicial complex $K$ is also a vertex of $\operatorname{Sd} K$, we see that

$$
\lambda^{\prime} \circ \mathrm{Sd}=\lambda, \quad \mu^{\prime} \circ \mathrm{Sd}=\mu .
$$

In the sequel we shall construct several natural transformations by aid of the acyclic model theorem, [S] 4.3.3. To this end, we fix some more notation. Let $\mathcal{M}_{\mathcal{U}} \subset \mathrm{Ob} \mathcal{C}_{\mathcal{U}}$ be a set of representatives for the $\mathcal{C}_{\mathcal{U}}$-isomorphism classes of the complexes $\bar{s}, s$ a simplex of $K\left(\mathcal{U}_{H}\right)$ or $K\left(\mathcal{U}_{H}^{\prime}\right), H \leq G$. Further, let $\mathcal{M}_{\mathcal{U}}^{\prime} \subset \mathrm{Ob} \mathcal{C}_{\mathcal{U}}^{\prime}$ be a set of representatives for the $\mathcal{C}_{\mathcal{U}}^{\prime}$-isomorphism classes of the complexes $\bar{\sigma}, \sigma$ a simplex of $K^{\prime}\left(\mathcal{U}_{H}\right)$ or $K^{\prime}\left(\mathcal{U}_{H}^{\prime}\right), H \leq G$. Also, choose the sets $\mathcal{N}_{\mathcal{U}} \subset \mathrm{Ob} \mathcal{D}_{\mathcal{U}}$ and $\nu_{\mathcal{U}}^{\prime} \subset \mathrm{Ob} \mathcal{D}_{\mathcal{U}}^{\prime}$ analogously.

Proposition 3.5. There are natural transformations $p: \Phi^{\prime} \rightarrow \Phi \circ P$ and $p: \Psi^{\prime} \rightarrow \Psi \circ P$.

Proof. By the acyclic model theorem, it is enough to show that the functors $\Phi^{\prime}$ and $\Psi^{\prime}$ are free with models $\mathcal{M}_{\mathcal{U}}^{\prime}$ and $\mathcal{N}_{\mathcal{U}}^{\prime}$, respectively, and the functors $\Phi \circ P$ and $\Psi \circ P$ are acyclic on the models $\mathcal{M}_{\mathcal{U}}^{\prime}$ and $\mathcal{N}_{\mathcal{U}}^{\prime}$, respectively.

The freeness of $\Phi^{\prime}$ and $\Psi^{\prime}$ is clear by construction. To prove the acyclicity of $\Phi \circ P$, let $\bar{\sigma} \in \mathcal{M}_{\mathcal{U}}^{\prime}$. If $s \in \sigma$ is the largest vertex of $\sigma$ (with respect to inclusion), then $(\Phi \circ P)(\bar{\sigma})=C_{*}(\bar{s}) \in C^{a}(A b)$ which is, of course, acyclic. The acyclicity of $\Psi \circ P$ is proved similarly. $\square$

We can form the following composite natural transformations:

$$
\begin{aligned}
& \Phi \stackrel{\text { sd }}{\longrightarrow} \Phi^{\prime} \circ \mathrm{Sd} \stackrel{(\mathrm{Sd})^{*}(p)}{\longrightarrow} \Phi \circ P \circ \mathrm{Sd} \\
& \Phi^{\prime} \stackrel{p}{\longrightarrow} \Phi \circ P \stackrel{P^{*}(\mathrm{sd})}{\longrightarrow} \Phi^{\prime} \circ \operatorname{Sd} \circ P \\
& \Psi \stackrel{\text { sd }}{\longrightarrow} \Psi^{\prime} \circ \mathrm{Sd} \stackrel{(\mathrm{Sd})^{*}(p)}{\longrightarrow} \Psi \circ P \circ \mathrm{Sd} \\
& \Psi^{\prime} \stackrel{p}{\longrightarrow} \Psi \circ P \stackrel{P^{*}(\mathrm{sd})}{\longrightarrow} \Psi^{\prime} \circ \mathrm{Sd} \circ P .
\end{aligned}
$$

Further, if $K \in \mathcal{C}_{\mathcal{U}}$, then $K \subset P(\operatorname{Sd} K)$, and this defines a natural transformation $I: \Phi \rightarrow \Phi \circ P \circ \operatorname{Sd}$. In the same way we obtain natural transformations $I: \Phi^{\prime} \rightarrow$ $\Phi^{\prime} \circ \operatorname{Sd} \circ P, I: \Psi \rightarrow \Psi \circ P \circ \operatorname{Sd}$ and $I: \Psi^{\prime} \rightarrow \Psi^{\prime} \circ \operatorname{Sd} \circ P$.

Proposition 3.6. There are natural chain homotopies

$$
(\mathrm{Sd})^{*}(p) \circ \mathrm{sd} \simeq I, \quad P^{*}(\mathrm{sd}) \circ p \simeq I .
$$

Proof. For example, the assertion concerning the natural transformations between $\Phi$ and $\Phi \circ P \circ S d$ follows from the trivial observation that $\Phi$ is free with models $\mathcal{M}_{\mathcal{U}}$ and $\Phi \circ P \circ \mathrm{Sd}$ is acyclic on the same models. $\square$ 
Proof of Proposition 2.4. We prove that the chain map sd: $\underline{C}_{*}\left(\mathcal{U}, \mathcal{U}^{\prime}\right) \rightarrow$ $\underline{C}_{*}^{\prime}\left(\mathcal{U}, \mathcal{U}^{\prime}\right)$ is a chain homotopy; the case of sd: $\underline{D}_{*}\left(\mathcal{U}, \mathcal{U}^{\prime}\right) \rightarrow \underline{D}_{*}^{\prime}\left(\mathcal{U}, \mathcal{U}^{\prime}\right)$ is similar.

To construct a homotopy inverse $p: \underline{C}_{*}^{\prime}\left(\mathcal{U}, \mathcal{U}^{\prime}\right) \rightarrow \underline{C}_{*}\left(\mathcal{U}, \mathcal{U}^{\prime}\right)$ of sd, we observe that the chain maps

$$
\begin{aligned}
& p: \quad \Phi^{\prime}\left(K^{\prime}\left(\mathcal{U}_{H}\right)\right) \quad \longrightarrow \Phi\left(P\left(K^{\prime}\left(\mathcal{U}_{H}\right)\right)\right)=\Phi\left(K\left(\mathcal{U}_{H}\right)\right) \\
& \text { II } \\
& \underline{C}_{*}^{\prime}(\mathcal{U})(G / H) \quad \underline{C}_{*}(\mathcal{U})(G / H)
\end{aligned}
$$

for $H \leq G$ are compatible with $G$-maps $G / H_{1} \rightarrow G / H_{2}$ and hence determine a chain map $p: \underline{C}_{*}^{\prime}(\mathcal{U}) \rightarrow \underline{C}_{*}(\mathcal{U})$. In the same way we get a chain map $p: \underline{C}_{*}^{\prime}\left(\mathcal{U}^{\prime}\right) \rightarrow$ $\underline{C}_{*}\left(\mathcal{U}^{\prime}\right)$, and the left hand square in the diagram

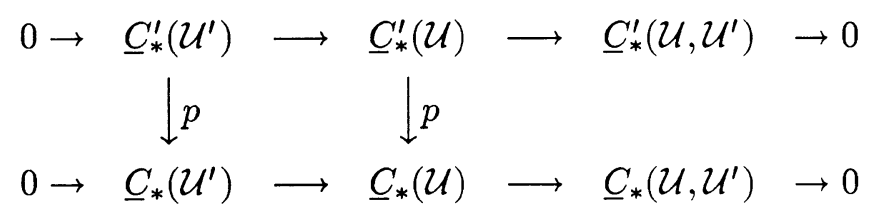

commutes, because the horizontal arrows are induced by morphisms of $\mathcal{C}_{\mathcal{U}}^{\prime}$. Thus the required $p: \underline{C}_{*}^{\prime}\left(\mathcal{U}, \mathcal{U}^{\prime}\right) \rightarrow \underline{C}_{*}\left(\mathcal{U}, \mathcal{U}^{\prime}\right)$ exists. Proposition 3.6 then allows us to construct chain homotopies $p \circ \mathrm{sd} \simeq \mathrm{id}$ and $\operatorname{sdop} \simeq \mathrm{id}$, proving that $p$ is a homotopy inverse of sd. $\square$

Proposition 3.7. There are natural transformations $\alpha: \Phi^{\prime} \rightarrow \Psi^{\prime} \circ \operatorname{Sd} \circ \lambda^{\prime}$ and $\beta: \Psi^{\prime} \rightarrow \Phi^{\prime} \circ \mathrm{Sd} \circ \mu^{\prime}$.

Proof. Because $\Phi^{\prime}$ and $\Psi^{\prime}$ are free with models $\mathcal{M}_{\mathcal{U}}^{\prime}$ and $\mathcal{N}_{\mathcal{U}}^{\prime}$, respectively, it is sufficient to show that $\Psi^{\prime} \circ \operatorname{Sd} \circ \lambda^{\prime}$ and $\Phi^{\prime} \circ \operatorname{Sd} \circ \mu^{\prime}$ are acyclic on the same models. We consider only $\Psi^{\prime} \circ \mathrm{Sd} \circ \lambda^{\prime}$.

Let $\sigma$ be a simplex of $K^{\prime}\left(\mathcal{U}_{H}\right), H \leq G$ (the case $\sigma \in K^{\prime}\left(\mathcal{U}_{H}^{\prime}\right)$ is similar). Then $\lambda^{\prime}(\bar{\sigma})=\lambda(s)$, where $s$ is the smallest vertex of $\sigma$. Write $s=$ $\left\{x_{0}, \ldots, x_{n}\right\}$ and pick $z \in U_{x_{0}} \cap \cdots \cap U_{x_{n}} \cap X^{H}$. Because $\left\{y_{0}, \ldots, y_{m}\right\} \subset X^{H}$ is a simplex of $\lambda(s)$ if and only if $\left\{y_{0}, \ldots, y_{m}\right\} \subset U_{x_{0}} \cap \cdots \cap U_{x_{n}}$, the formula $\left(y_{0}, \ldots, y_{m}\right) \mapsto\left(z, y_{0}, \ldots, y_{m}\right)$ defines a chain contraction of $C_{*}(\lambda(s)) \in C^{a}(A b)$. Because sd: $C_{*}(\lambda(s)) \rightarrow C_{*}(\operatorname{Sd} \lambda(s))=\left(\Psi^{\prime} \circ \operatorname{Sd} \circ \lambda^{\prime}\right)(\bar{\sigma})$ is a chain homotopy equivalence, $\left(\Psi^{\prime} \circ \mathrm{Sd} \circ \lambda^{\prime}\right)(\bar{\sigma})$ is acyclic. $\square$

Now we can form the following natural transformations:

$$
\begin{aligned}
& \Phi^{\prime} \stackrel{\alpha}{\longrightarrow} \Psi^{\prime} \circ \operatorname{Sd} \circ \lambda^{\prime} \stackrel{\left(\operatorname{Sd} \circ \lambda^{\prime}\right)^{*}(\beta)}{\longrightarrow} \Phi^{\prime} \circ \operatorname{Sd} \circ \mu^{\prime} \circ \operatorname{Sd} \circ \lambda^{\prime}=\Phi^{\prime} \circ \operatorname{Sd} \circ \mu \circ \lambda^{\prime} \\
& \Psi^{\prime} \stackrel{\beta}{\longrightarrow} \Phi^{\prime} \circ \operatorname{Sd} \circ \mu^{\prime} \stackrel{\left(\operatorname{Sd} \circ \mu^{\prime}\right)^{*}(\alpha)}{\longrightarrow} \Psi^{\prime} \circ \operatorname{Sd} \circ \lambda^{\prime} \circ \operatorname{Sd} \circ \mu^{\prime}=\Psi^{\prime} \circ \operatorname{Sd} \circ \lambda \circ \mu^{\prime} .
\end{aligned}
$$

Further, let $I: \Phi^{\prime} \rightarrow \Phi^{\prime} \circ \operatorname{Sd} \circ \mu \circ \lambda^{\prime}$ and $I: \Psi^{\prime} \rightarrow \Psi^{\prime} \circ \operatorname{Sd} \circ \lambda \circ \mu^{\prime}$ be the natural transformations determined by the inclusions $K^{\prime} \hookrightarrow \operatorname{Sd}\left(\mu\left(\lambda^{\prime}\left(K^{\prime}\right)\right)\right)$ and $L^{\prime} \hookrightarrow$ $\operatorname{Sd}\left(\lambda\left(\mu^{\prime}\left(L^{\prime}\right)\right)\right)$ for $K^{\prime} \in \mathcal{C}_{\mathcal{U}}^{\prime}$ and $L^{\prime} \in \mathcal{D}_{\mathcal{U}}^{\prime}$. 
Proposition 3.8. There are natural chain homotopies

$$
\left(\operatorname{Sd} \circ \lambda^{\prime}\right)^{*}(\beta) \circ \alpha \simeq I, \quad\left(\operatorname{Sd} \circ \mu^{\prime}\right)^{*}(\alpha) \circ \beta \simeq I .
$$

Proof. It is enough to show that the functors $\Phi^{\prime} \circ \operatorname{Sd} \circ \mu \circ \lambda^{\prime}$ and $\Psi^{\prime} \circ \operatorname{Sd} \circ \lambda \circ \mu^{\prime}$ are acyclic on the models $\mathcal{M}_{\mathcal{U}}^{\prime}$ and $\mathcal{N}_{\mathcal{U}}^{\prime}$, respectively. We consider only $\Phi^{\prime} \circ S d \circ \mu \circ$ $\lambda^{\prime}$, and leave the (similar) other case to the reader.

Let $\sigma$ be a simplex of $K^{\prime}\left(\mathcal{U}_{H}\right), H \leq G$, and $s=\left\{x_{0}, \ldots, x_{n}\right\}$ the smallest vertex of $\sigma$. Then $\lambda^{\prime}(\bar{\sigma})=\lambda(s)$, as noted in the proof of 3.7. Now $t=$ $\left\{z_{0}, \ldots, z_{q}\right\} \subset X^{H}$ is a simplex of $\mu(\lambda(s))$, if and only if there is a vertex $y \in \lambda(s)$ (i.e. $\left.y \in U_{x_{0}} \cap \cdots \cap U_{x_{n}} \cap X^{H}\right)$ such that $y \in U_{z_{0}} \cap \cdots \cap U_{z_{q}} \cap X^{H}$; this is clearly equivalent to the condition $s \cup t \in K\left(\mathcal{U}_{H}\right)$ (i.e. $U_{x_{0}} \cap \cdots \cap U_{x_{n}} \cap U_{z_{0}} \cap \cdots \cap U_{z_{q}} \cap X^{H}$ $\neq \emptyset)$. Hence the formula $\left(z_{0}, \ldots, z_{q}\right) \mapsto\left(x_{0}, z_{0}, \ldots, z_{q}\right)$ defines a chain contraction of $C_{*}(\mu(\lambda(s))) \in C^{a}(A b)$, and so $\left(\Phi^{\prime} \circ \operatorname{Sd} \circ \mu \circ \lambda^{\prime}\right)(\bar{\sigma})=C_{*}(\operatorname{Sd}(\mu(\lambda(s)))) \simeq$ $C_{*}(\mu(\lambda(s)))$ is acyclic.

Assume now that $\mathcal{U}$ is a refinement of another $G$-covering $\mathcal{V}$ of $X$. If $H \leq G$, then every subcomplex $K^{\prime}$ of $K^{\prime}\left(\mathcal{U}_{H}\right)$ (or $K^{\prime}\left(\mathcal{U}_{H}^{\prime}\right)$ ) can also be regarded as a subcomplex of $K^{\prime}\left(\mathcal{V}_{H}\right)$ (or $K^{\prime}\left(\mathcal{V}_{H}^{\prime}\right)$ ). This defines a functor $j: \mathcal{C}_{\mathcal{U}}^{\prime} \rightarrow \mathcal{C}_{\mathcal{V}}^{\prime}$, and there is an analogous functor $j: \mathcal{D}_{\mathcal{U}}^{\prime} \rightarrow \mathcal{D}_{\mathcal{V}}^{\prime}$, too. There are also obvious natural transformations

$$
\varrho: \Phi_{\mathcal{U}}^{\prime} \rightarrow \Phi_{\mathcal{V}}^{\prime} \circ j, \quad \tau: \Psi_{\mathcal{U}}^{\prime} \rightarrow \Psi_{\mathcal{V}}^{\prime} \circ j
$$

for example, if $K^{\prime} \in \mathcal{C}_{\mathcal{U}}^{\prime}$, then $\varrho\left(K^{\prime}\right)$ is simply the identity of $C_{*}\left(K^{\prime}\right)$.

We consider the following composite natural transformations:

$$
\begin{gathered}
\Phi^{\prime} \stackrel{\alpha}{\longrightarrow} \Psi_{\mathcal{U}}^{\prime} \circ \operatorname{Sd} \circ \lambda^{\prime} \stackrel{\left(\operatorname{Sd} \circ \lambda^{\prime}\right)^{*}(\tau)}{\longrightarrow} \Psi_{\mathcal{V}}^{\prime} \circ j \circ \operatorname{Sd} \circ \lambda^{\prime} \\
\Phi_{\mathcal{U}}^{\prime} \stackrel{\varrho}{\longrightarrow} \Phi_{\mathcal{V}}^{\prime} \circ j \stackrel{j^{*}(\alpha)}{\longrightarrow} \Psi_{\mathcal{V}}^{\prime} \circ \operatorname{Sd} \circ \lambda^{\prime} \circ j=\Psi_{\mathcal{V}}^{\prime} \circ j \circ \operatorname{Sd} \circ \lambda^{\prime} .
\end{gathered}
$$

Proposition 3.9. There is a natural chain homotopy

$$
\left(\operatorname{Sd} \circ \lambda^{\prime}\right)^{*}(\tau) \circ \alpha \simeq j^{*}(\alpha) \circ \varrho .
$$

Proof. The functor $\Phi_{\mathcal{U}}^{\prime}$ is free with models $\mathcal{M}_{\mathcal{U}}^{\prime}$, and $\Psi_{\mathcal{V}}^{\prime} \circ j \circ \mathrm{Sd} \circ \lambda^{\prime}$ is acyclic on the same models. $\square$

Proof of Proposition 2.5. The natural transformations $\alpha$ and $\beta$ of 3.7 allow us to construct chain maps $\alpha: \underline{C}_{*}^{\prime}\left(\mathcal{U}, \mathcal{U}^{\prime}\right) \rightarrow \underline{D}_{*}^{\prime}\left(\mathcal{U}, \mathcal{U}^{\prime}\right)$ and $\beta: \underline{D}_{*}^{\prime}\left(\mathcal{U}, \mathcal{U}^{\prime}\right) \rightarrow$ $\underline{C}_{*}^{\prime}\left(\mathcal{U}, \mathcal{U}^{\prime}\right)$ in the same way as the chain map $p: \underline{C}_{*}^{\prime}\left(\mathcal{U}, \mathcal{U}^{\prime}\right) \rightarrow \underline{C}_{*}\left(\mathcal{U}, \mathcal{U}^{\prime}\right)$ was contructed in the proof of 2.4. From 3.9 it then follows that $\alpha$ is compatible with refinement up to chain homotopy, and 3.8. shows that $\beta$ is a chain homotopy inverse of $\alpha$. व 


\section{Appendix}

In this appendix we prove Proposition 1.2. Let $(X, A)$ be a $G$-pair and $m \in \mathcal{C}_{G}$ a coefficient system. There are exact sequences

$$
\begin{aligned}
0 & \rightarrow \underset{\mathcal{u}}{\lim } \operatorname{Hom}_{\mathcal{C}_{G}}\left(\underline{D}_{*}\left(\mathcal{U}, \mathcal{U}^{\prime}\right), m\right) \rightarrow \underline{\lim } \operatorname{Hom}_{\mathcal{C}_{G}}\left(\underline{D}_{*}(\mathcal{U}), m\right) \\
\rightarrow & \underset{\lim }{\operatorname{lom}} \operatorname{Hom}_{\mathcal{C}_{G}}\left(\underline{D}_{*}\left(\mathcal{U}^{\prime}\right), m\right), \\
& \quad 0 \rightarrow \bar{C}_{G}^{*}(X, A ; m) \rightarrow \bar{C}_{G}^{*}(X ; m) \rightarrow \bar{C}_{G}^{*}(A ; m) ;
\end{aligned}
$$

the former is a consequence of the left exactness of $\operatorname{Hom}_{\mathcal{C}_{G}}$ and exactness of $\stackrel{\lim }{\longrightarrow}$, while the latter follows from Definition $1.1 \mathrm{in}[\mathrm{H}]$. Therefore it is enough to show that

$$
\bar{C}_{G}^{*}(X ; m) \cong \underset{\mathcal{U}}{\lim _{\longrightarrow}} \operatorname{Hom}_{\mathcal{C}_{G}}\left(\underline{D}_{*}(\mathcal{U}), m\right)
$$

and

$$
\bar{C}_{G}^{*}(A ; m) \cong \underset{\mathcal{u}}{\lim } \operatorname{Hom}_{\mathcal{C}_{G}}\left(\underline{D}_{*}\left(\mathcal{U}^{\prime}\right), m\right)
$$

(provided that the isomorphisms are functorial enough). In fact it suffices to prove the first isomorphism, for the second is a special case of the first by the next lemma:

Lemma A.1. Any $G$-covering $\mathcal{V}$ of $A$ is of the form $\mathcal{U}^{\prime}$ for some $G$-covering $\mathcal{U}$ of $X$.

Proof. Given $\mathcal{V}$, a desired $\mathcal{U}$ can be constructed as follows: Pick a representative $x \in A$ for each $G$-orbit of $A$ and choose an open $U_{x} \subset X$ for every $x$ so that $U_{x}$ is $G_{x}$-invariant and $U_{x} \cap A=V_{x}$. If $y=g x \in A, g \in G$, let $U_{y}=g U_{x}$. In this way $U_{y}$ is defined for every $y \in A$. For $y \in X \backslash A$, choose $U_{y}$ arbitrarily to satisfy $y \in U_{y}$ and $g U_{y}=U_{g y}, g \in G$. $\square$

In $[\mathrm{H}]$ we defined $\bar{C}_{G}^{n}(X ; m)=C_{G}^{n}(X ; m) / C_{G, 0}^{n}(X ; m)$, where $C_{G, 0}^{n}(X ; m) \subset$ $C_{G}^{n}(X ; m)$ is the subgroup of locally zero cochains; a cochain $c \in C_{G}^{n}(X ; m)$ is locally zero, if there is a $G$-covering $\mathcal{U}$ of $X$ such that

$$
\begin{aligned}
c(\varphi)=0 & \text { for } \varphi \in V_{n}(X), \quad t(\varphi)=H, \\
& \text { if }\left\{\varphi_{0}(e H), \ldots, \varphi_{n}(e H)\right\} \subset U_{x} \text { for some } x \in X
\end{aligned}
$$

(here we have used the notation of $[\mathrm{H}]$ ).

Remark A.3. When defining locally zero cochains in $[\mathrm{H}]$, we used a more general notion of a $G$-covering than in this paper. These different notions lead to the same concept of locally zero cochains, however, because any open $G$-covering $\mathcal{W}$ of $X$ in the sense of [H] has a refining $G$-covering $\mathcal{U}=\left\{U_{x} \mid x \in X\right\}$ in the sense of this paper; such a $\mathcal{U}$ can be obtained by an orbitwise construction as in A.1. 
Let us also consider the following condition for a cochain $c \in C_{G}^{n}(X ; m)$ and a $G$-covering $\mathcal{U}$ of $X$ :

$$
\begin{aligned}
c(\varphi)=0 & \text { for } \varphi \in V_{n}(X), \quad t(\varphi)=H, \\
& \text { if }\left\{\varphi_{0}(e H), \ldots, \varphi_{n}(e H)\right\} \subset U_{x} \text { for some } x \in X^{H} .
\end{aligned}
$$

Denote ${ }^{\prime} C_{G, 0}^{n}(X ; m)=\left\{c \in C_{G}^{n}(X ; m) \mid c\right.$ satisfies A.4 for some $\left.\mathcal{U}\right\}$.

Lemma A.5. We have

$$
\underset{\mathcal{U}}{\lim _{\longrightarrow}} \operatorname{Hom}_{\mathcal{C}_{G}}\left(\underline{D}_{n}(\mathcal{U}), m\right) \cong C_{G}^{n}(X ; m) /{ }^{\prime} C_{G, 0}^{n}(X ; m) .
$$

Proof. Any $\varphi=\left(\varphi_{0}, \ldots, \varphi_{n}\right) \in V_{n}(X)$ determines an $(n+1)$-tuple $\left(x_{0}, \ldots, x_{n}\right) \in\left(X^{H}\right)^{n+1}, H=t(\varphi)$, by $x_{i}=\varphi_{i}(e H)$, and conversely. From this it follows that if $\mathcal{U}$ is a $G$-covering of $X$ and we denote

$$
\begin{aligned}
V_{n}^{\prime}(X, \mathcal{U})=\{\varphi & \in V_{n}(X) \mid\left\{\varphi_{0}(e H), \ldots, \varphi_{n}(e H)\right\} \\
& \left.\subset U_{x} \text { for some } x \in X^{H}, H=t(\varphi)\right\}
\end{aligned}
$$

and $C_{n}^{\prime}(\mathcal{U})=$ free abelian group with basis $V_{n}^{\prime}(X, \mathcal{U})$, then

$$
\begin{aligned}
& \operatorname{Hom}_{\mathcal{C}_{G}}\left(\underline{D}_{n}(\mathcal{U}), m\right) \cong \\
& \left\{u: C_{n}^{\prime}(\mathcal{U}) \rightarrow \bigoplus_{H \leq G} m(G / H) \mid u\right. \text { is a homomorphism; } \\
& u(\varphi) \in m(G / t(\varphi)) \text { for each } \varphi \in V_{n}^{\prime}(X, \mathcal{U}) ; \\
& \text { if } \alpha: G / K \rightarrow G / t(\varphi) \text { is a } G-\operatorname{map} \text {, then } u(\varphi \circ \alpha)=m(\alpha)(u(\varphi))\} \text {. }
\end{aligned}
$$

The assertion of the lemma is then verified in the same way as Lemma $4.1 \mathrm{in}[\mathrm{H}]$. $\square$

The proof of the identity $\bar{C}_{G}^{n}(X ; m) \cong \lim _{\longrightarrow} \operatorname{Hom}_{\mathcal{C}_{G}}\left(\underline{D}_{n}(\mathcal{U}), m\right)$ is now completed by the lemma below:

Lemma A.6. Let $c \in C_{G}^{n}(X ; m)$ and let $\mathcal{U}$ be a $G$-covering of $X$.

i) If $c$ satisfies A.2 for $\mathcal{U}$, then $c$ satisfies $\mathrm{A} .4$ for $\mathcal{U}$;

ii) if $c$ satisfies A.4 for $\mathcal{U}$, then there is a refinement $\mathcal{V}$ of $\mathcal{U}$ such that $c$ satisfies A.2 for $\mathcal{V}$.

Proof. Part i) is trivial. As for ii), a required $\mathcal{V}$ can be constructed as follows: Pick a representative $x \in X$ from every $G$-orbit of $X$; for each such an $x$ choose an open $G_{x}$-invariant neighborhood $V_{x}$ such that $V_{x} \subset U_{x}$ and $V_{x} \cap X^{H}=\emptyset$, if $H \leq G$ and $x \notin X^{H}$ (recall that $X^{H}$ is closed, because $X$ is Hausdorff); if $y=g x, g \in G$, let $U_{y}=g U_{x}$. ᄆ 


\section{References}

[B] Bredon, G.E.: Equivariant cohomology theories. - Lecture Notes in Mathematics 34. Springer-Verlag, New York, 1967.

[D] Dowker, C.H.: Homology groups of relations. - Ann. of Math. 56, 1952, 84-95.

[E-St] Eilenberg, S., and N. Steenrod: Foundations of algebraic topology. - Princeton University Press, Princeton, 1952.

[G] Godement, R.: Topologie algébrique et théorie des faisceaux. - Hermann \& C ${ }^{\text {ie }}$, Paris, 1958.

[H] Honkasalo, H.: Equivariant Alexander-Spanier cohomology. - Math. Scand. 63, 1988, 179-195.

[S] Spanier, E.H.: Algebraic topology. - McGraw-Hill, New York-Toronto-London, 1966.

University of Helsinki

Department of Mathematics

Hallituskatu 15

SF-00100 Helsinki

Finland

Received 12 March 1990 